REVISTA DE DERECHO UNED, NÚM. 17, 2015

\title{
NEUROCIENCIA, DERECHO Y DERECHOS HUMANOS
}

\author{
NEUROSCIENCE, LAW AND HUMAN RIGHTS
}

\begin{abstract}
Manuel Ruiz Martínez-Cañavate
Investigador doctorando en la Facultad de Derecho UNED y Fiscal de la Fiscalía Provincial de Granada (Sección Territorial de Motril)
\end{abstract}

Resumen: El avance de las neurociencias en las últimas décadas ha desencadenado importantes consecuencias en el terreno de la medicina y la biología. De un modo paralelo, el desarrollo de las aplicaciones neurotecnológicas abre importantes vías de relación con el Derecho, en particular en los ámbitos penal y procesal. Los efectos de tal relación pueden llevar al replanteamiento de nociones clave como la libertad, la voluntad, la responsabilidad, o el castigo. En el terreno probatorio, tales avances exigen valorar la viabilidad y eticidad de los denominados medios de prueba cerebrales. Ante tales progresos, la Neuroética nació como ciencia en el Congreso de San Francisco de 2002, con una vocación interdisciplinar. La propia definición y virtualidad de la Neuroética significa para autores destacados en el campo de la filosofía moral y jurídica la consagración de la posibilidad de una ética universal basada en el cerebro humano. Adela Cortina matiza que tal afirmación sólo representaría una ética con fundamento cerebral.

Palabras clave: Neurociencias, neuroética, derechos humanos, desafíos éticos, desafíos jurídicos

Abstract: Last decades neuroscientific progress has developed significant consequences in the field of medicine and biology. Next to that, neurotechnologic applications development open important ways of relationship with law field, specially, in criminal and trial law. 
Such effects could redefine key words such as freedom, will, liability or punishment. In the probe law field, neuroscientific developments mean to value the viability and ethic character of the named neural probe measures. Because of that, Neuroethics bore in 2002 in San Francisco's Congress, with a interdisciplinary vocation. Neuroethics' definition and its effectiveness mean, for recognized authors in Moral and Law Philosophy the reality of a possibility of an Ethic based in the human brain. Adela Cortina condition such conclusion in the sense that just would represent an Ethic with a brain foundations.

Keywords: Neurosciences, neuroethics, human rights, ethics challenges, juridical challenges

Recepción original: 08/06/2015

Aceptación original: 13/07/2015

A NANI, por ser todo

Sumario: I. Introducción general. II. Introducción a la neurociencia: A. Planteamiento de partida: el enfoque neural. B. Definiciones. Contenidos. Objetivos. III. Desafíos ético-jurídicos nacidos de la evolución neurocientífica. A. Identificación de los desafíos. Autores relevantes. B. La inevitable conexión Neurociencias-Derecho Penal. IV. La neuroética en relación con los Derechos Humanos. V. Aportación de la neuroética a la construcción de una ética universal. del «ES» cerebral al «DEBE» moral. VI. Conclusiones.

\section{INTRODUCCIÓN GENERAL}

1. Las últimas décadas del siglo $\mathrm{Xx}$ supusieron un verdadero giro copernicano con los estudios sobre ADN, hasta el punto de hablar de revolución neurotecnológica. Para Jonathan MORENO, la primera década del siglo XXI ha sido el germen de un nuevo cataclismo en el plano de la investigación cerebral. Sobre la base de los descubrimientos científicos de la medicina cerebral y la neurobiología, en el presente trabajo nos proponemos ensanchar la mirada; abrir las perspectivas y explorar las repercusiones filosóficas que las nuevas técnicas de percepción de la actividad cerebral aportan. En el camino que iniciamos, nos proponemos sobrevolar los principales hitos institucionales que han determinado la aparición de una nueva ciencia, a la que el Congreso de San Francisco en 2002 sirvió para otorgar carta de naturaleza: la neuroética. El éxito de la definición -voz "Neuroethics»-, y la estela mediática que ha seguido la irrupción en la órbita científica, ha propiciado importantes movimientos en el 
ámbito institucional y en de la divulgación. En cuanto al primero, tras el Congreso de la Society for Neuroscience -verdadera precursora de los pasos venideros-, en 2006 se fundó -con una vocación más específica- la Neuroethics Society. En el segundo de los aspectos, los editoriales de las prestigiosas publicaciones Science y Nature han girado con llamativa frecuencia sobre cuestiones neuroéticas. Sin embargo, desde marzo de 2008, la editorial Springer viene publicando la revista Neuroethics, auténtico referente y escaparate en la nueva disciplina. Asimismo, y desde la órbita académica, en 2007 se creó, bajo los auspicios de la Universidad de British Columbia de Vancouver el «National Core for Neuroethics». Dos años más tarde, en 2009, abrió sus puertas «The Wellcome Center for Neuroethics» en el seno de la Universidad de Oxford.

2. La afortunada expresión empleada ilustrativamente por Steven ROSE pone de manifiesto que en la «era del cerebro», será éste el verdadero eje alrededor del que gire el universo y los modos técnicos de aproximarse o percibir la realidad. De este modo, aunque nos sobrevuele el interrogante de si la sociedad venidera será post-humana -en expresión acuñada por Adela Cortina-, la realidad será necesariamente neural o no será. Esta nueva visión de la realidad del ser humano y sus interrogantes desmadejará múltiples aplicaciones, por lo que será objetivo de este estudio desentrañar aquellas consecuencias que incidan en los planos éticos y jurídicos, con particular atención a la dimensión iusfundamental.

3. Desde un enfoque necesariamente interdisciplinar el derecho se adentra en un terreno prácticamente inexplorado pero destinado a ser fuente de riqueza y de actitudes futuras constructivas. En cuanto que el Derecho contempla los avances técnicos y los descubrimientos de la Neurociencia, un nuevo fenómeno comienza a despegar desde la aceptación de los propios límites. Éste es uno de los puntos de partida que suscitará el desarrollo de las ideas de este proyecto: el Derecho no es compartimento científico estanco y necesita beber de otras fuentes, hasta el punto que esta opción comprobaremos cómo para algunos es una necesidad y, para otros, pura comprensión de la única realidad posible. En suma, la mirada estupefacta del Derecho habrá de transformarse en una visión crítica en salvaguarda del tesoro de los Derechos Humanos en juego. Es ésta otra dimensión de la Neuroética, la de la perspectiva ética de los avances y procedimientos neurocientíficos, en colisión con las posiciones más posibilistas.

4. Pero la Neuroética, concebida tanto como un conjunto de principios éticos como vértice estratégico de interrogantes éticos y meto-

(C) UNED. Revista de Derecho UNED, núm. 17, 2015 
dológicos -es decir, como realidad y desafío al tiempo-, trasciende notablemente el ámbito y las potenciales de una bioética aplicada más. Algunos atisban en ella una clave de bóveda desde la que ordenar el universo entero, desde el papel nuclear del cerebro como centro de todo el desarrollo ético de la humanidad. ¿Será la neural la piedra filosofal que esconda los secretos más oscuros para la comprensión del proceso ético humano? ¿Radicará en un juego de neurotransmisores el equilibrio y funcionamiento de la decisión moral de toda persona? Avancemos. Reflexionaremos sobre las consecuencias inmediatas que para el Derecho y las ciencias sociales tiene la visualización directa del fluir cerebral. ¿Se tratará de mera comprobación de los datos que se presentan ante nuestra atenta mirada o más bien se antoja imprescindible una labor de interpretación de esta realidad percibida? Una vez más, la respuesta no puede ser unívoca y precisará de las dos, verificación y hermenéutica.

5. Y, durante la búsqueda, ¿qué sucederá si al tiempo que identificamos las claves del razonar humano universal encontramos que éstas colisionan como derechos fundamentales, como los recogidos en la Declaración Universal de los Derechos Humanos? Parece claro que la nueva Bioética aplicada, la Neuroética sólo se consagrará en cuanto sus resultados sean compatibles con los Derechos Humanos. Neurociencia y Neuroética han de superar el ineludible filtro de la legitimidad, la cuestión más vieja del Derecho. Por lo tanto, estamos en los albores de una nueva era, la "era del cerebro», de manera que igual que en otro tiempo los estudios de $\mathrm{ADN}$ trajeron consigo la revolución tecnológica, probablemente sólo nos encontremos ante los movimientos incipientes de una nueva revolución, que ya será analizada desde la observación de las conexiones neurales y neurotransmisores. En la era de la revolución neurológica, el Derecho no es una etapa más en el camino de la Neurociencia a la Neuroética.

\section{INTRODUCCIÓN A LA NEUROCIENCIA}

\section{Planteamiento de partida: el enfoque neural}

Este estudio nace en una clave dinámica de aproximación del derecho a esa rama de la ciencia médica que es la Neurociencia, al tiempo que una toma de temperatura con el enfoque metodológico que supone la denominada Neuroética. Desde las claves de este binomio se pretende un replanteamiento de algunas de las preguntas 
humanas clásicas acerca de la naturaleza y el comportamiento de la persona. En consecuencia, toda profundización en el tema no puede soslayar la evidente interdisciplinariedad que marcó el nacimiento de la neurociencia como factor determinante en el protagonismo creciente del enfoque neuroético ${ }^{1}$. Hoy, partiendo de la neurociencia como estudio interdisciplinar del sistema nervioso ${ }^{2}$, proliferan neologismos ${ }^{3}$ tales como «neurobiología», "neuroimagen», «neuroética», «neuromoral», «neurofilosofía», «neuromarketing» o «neuroderecho». Su éxito futuro como términos que designen particulares segmentos del área de conocimiento neural probablemente no dependa tanto de lo acertado de su elección como de la autonomía que alcance como rama de la ciencia el objeto definido. Al respecto la profesora Narváez Mora diferencia neurociencia de neurobiología -en ocasiones empleados como sinónimos-, desde el prisma que la referencia a la neurobiología excluye materias como la neuroimagen, ciñéndose al mero estudio de la biología del sistema nervioso. En consecuencia, desde la perspectiva dinámica que metodológicamente asumimos, la neurociencia la definiremos como aquella rama de la ciencia médica responsable del estudio interdisciplinar del sistema nervioso.

Ya hemos adelantado que la perspectiva neurobiológica se deja fuera las modernas aplicaciones de la neuroimagen, entendida ésta como el acervo de técnicas que facultan para la obtención de imágenes del cerebro. Tal vez en ella se hallen algunas de las respuestas a las cuestiones suscitadas en las líneas antecedentes de este trabajo. Ellas serán el eslabón a nuestro primer bloque de desarrollo temático. Las próximas líneas abundarán por tanto en las posibilidades técnicas, principalmente de escáner tomográfico por computación y de la resonancia magnética funcional. Su desarrollo histórico camina de la mano con la visión de nuestro cerebro y, más importante aún, con la visión de nuestra actividad cerebral. De la acción humana nos interesan sus dimensiones ética y jurídica. Con el desarrollo de la neuroimagen, hoy somos más transparentes ¿lo son también nuestras decisiones éticas?

${ }^{1}$ SÁNCHEZ-MIGALLÓN, S. «La ambigüedad de la neuroética», Murcia, 2011 [ conferencia pronunciada el 21 de enero de 2011, publicado por la Fundación Bioética en la dirección http://www.bioeticas.org/?dst=neuroetica].

2 NARVÁEZ MORA, M. "Notas sobre neurociencia, filosofía y derecho», en Legal Theory and Philosophy, Working Papers Series n. ${ }^{\circ}$ 10, Universitat de Girona.

${ }^{3}$ SLACHEVSKY, A. «La neuroética: ¿Un neologismo infundado o una nueva disciplina?»Revista Chilena de Neuro-Psiquiatría, v. 45, 1, 2007, págs. 12-15 v. 45, 1, 2007, págs. 12-15. 


\section{Definiciones. Contenidos. Objetivos}

Ya nos hemos decantado por una definición sintética de neurociencia en tanto rama de la medicina responsable del estudio interdisciplinar del sistema nervioso. En esta línea los profesores SánchezMigallón y Giménez Amaya ${ }^{4}$ explican cómo el nacimiento de la neurociencia sólo se entiende desde la preocupación y aportación multidisciplinar de las diversas ciencias biológicas -junto a la psicología y psiquiatría- que se ocupan históricamente del sistema nervioso, en sus estadios normal y patológico. De modo que ese enfoque plural y perspectivista al sistema nervioso se configura como un factor determinante en la definición global de la neurociencia. Estos autores señalan otro elemento sustancial en la delimitación actual de la neurociencia, cual es que la evolución de la disciplina neurocientífica ha sido en perfecta sincronía con la bioética, coincidiendo temporalmente con los avances biotecnológicos más significativos y generando una relación significativamente armónica entre los objetivos de una y otra. De este modo, es posible trazar una definición de la neurociencia desde la aproximación a su contenido y ámbito visibles, de modo que la moderna neurociencia tendría por objeto arrojar luz a los siguientes hitos bioéticos: 1) momentos inicial y final de la vida humana; 2) Relaciones médico/paciente; y 3) experimentación animal.

En este sentido, Adela Cortina ${ }^{5}$ se hace eco de la pedagógica estructura de Jonsen, quien analiza las cuestiones nacidas en el primer congreso del que brotó la nueva ciencia neuroética, ubicándolas en tres planos. De este modo, siempre según Jonsen, el mapa neuroético comprende tres planos sistémicos:

- En el nivel tectónico se abordarán los interrogantes estructurales. En este campo tienen cabida las grandes interpretaciones acerca de la libertad humana o el reduccionismo.

- En el nivel geográfico se analizarán las cuestiones de epistemología o morfología, acerca de las formas de conocimiento.

- En el nivel local tendrá cabida la casuística del conflicto particular. Éste es el espacio donde cobra sentido la estrategia

${ }^{4}$ SÁNCHEZ-MIGALLÓN GRANADOS, S. y GIMÉNEZ AMAYA, J. M. "Neuroética» en FERNÁNDEZ LABASTIDA, F. y MERCADO, J. A. (editores) Philosophica: Enciclopedia filosófica on line: http://www.philosophica.info/archivo/20092008/voces/ neuroetica/Neuroetica.html.

${ }^{5}$ CORTINA, A. Neuroética y Neuropolitica: Sugerencias para la Educación Moral, Madrid, Ed. Tecnos, 2011, págs. 72-76. 
interdisciplinar propia de la neuroética aplicada, en el que se ventilarán, entre otros, la investigación con seres humanos, la responsabilidad criminal o la viabilidad de las técnicas de tratamiento cerebral.

Esta misma relación de sincronía con la bioética la identifica Neil Levy $^{6}$ en la Neuroética, a la que previamente define como «una reflexión ética sobre el uso de las diversas tecnologías y técnicas provenientes de la neurociencia, y de otras ciencias de la mente», pasando a subrayar su coincidencia teleológica con la bioética. Estas afirmaciones encuentran eco en algunos de los interrogantes suscitados acerca de la utilización de los denominados psicofármacos, el empleo forense de las técnicas de imaginería cerebral y la responsabilidad última de personas aquejadas de graves enfermedades psiquiátricas, latiendo en todos estos interrogantes la noción acerca de la naturaleza biológica de la moral. Interesante es asimismo la definición de neuroética que nos aporta Judy Illes ${ }^{7}$ en una aproximación teleológica en tanto "disciplina que alinea la exploración y el descubrimiento del conocimiento neurobiológico con los sistemas humanos de valores», para continuar la explicación aclarando que su vector de penetración es en «intersección con la ética biomédica en la medida en que (...) le conciernen las implicaciones éticas, legales y sociales de los descubrimientos de las investigaciones científicas, y con la naturaleza de la investigación misma». De manera que se concibe como una ciencia comprometida. De un modo más sintético y con propósito finalista, Gazzaniga ${ }^{8}$ aprecia que la neuroética «es o debería ser un intento de proponer una filosofía de la vida con un fundamento cerebral».

A los efectos de este estudio, nos interesarán los avances neurocientíficos en cuanto asumamos la existencia del campo conocido como neuroderecho o neuroley ${ }^{9}$. Es preciso retroceder a junio de 2002, fecha en que tuvo lugar en San Francisco el congreso patrocinado por la Dana Foundation, organizado por las Universidades de Stanford y California bajo el título «Neuroethics: Mapping the field ${ }^{10}$. Jugando con la metáfora, podemos afirmar que el terreno ya estaba abonado para el surgimiento de una nueva disciplina: la neuroética, y ello en el

${ }^{6}$ LEVY, N. Neuroethics, Cambridge University Press, Nueva York, 2007, págs. 286-28.

${ }^{7}$ ILLES, J. y BIRD, S. J. "A Modern Context for Ethics in Neuroscience», Trends in Neuroscience n. ${ }^{\circ} 29$, págs. 511.

${ }^{8}$ GAZZANIGA, M. S. El cerebro ético, Ed. Paidós, Barcelona, 2006.

${ }^{9}$ Sobre la relación de recíproca retroalimentación, CARRARA, A. «Neuroderecho, entendiendo la mente de los criminales», 2010 y RODRÍGUEZ, pág. «La neurociencia como defensa penal» en la sección Ciencia y Futuro de la edición del diario $A B C$ de 12 de marzo de 2007.

${ }^{10}$ MARCUS, S. J. Neuroethics. Mapping the Field, The Dana Press, New York 2002. 
contexto multidisciplinar ya atisbado para la neurociencia, a lo que cabía unir la inmensa proyección de futuro que guardaban sus relaciones con el mundo del derecho. De modo que el enfoque neural se impone como un criterio vertebrador de no pocas disciplinas científicas $^{11}$, particularmente la médica y la jurídica, de modo que de esta novedosa fusión surgirán importantes consecuencias y perspectivas de análisis. Es así, que la bioética del siglo XXI sólo se entiende conforme al patrón neurocientífico. En este contexto, se antoja evidente que los avances neurocientíficos caracterizados por un ritmo galopante en las últimas décadas precisarán de una disciplina autónoma que indague y controle las resonancias éticas de sus aportaciones. Ahí es donde aparece la neuroética como aquella área dentro de la neurociencia que se preocupa ampliamente del componente ético de los avances en medicina neural. Éste es nuestro punto de partida. En la publicación nacida del congreso de San Francisco, Marcus ya contempla una definición de corte analítico -con vocación programáticaque viene a describir el contenido potencial de la neuroética: «El estudio de las cuestiones éticas, legales y sociales que surgen cuando los hallazgos científicos sobre el cerebro son llevados a la práctica médica, a las interpretaciones legales y a las políticas sanitarias o sociales. Estos hallazgos están ocurriendo en campos que van desde la genética o la imagen cerebral hasta el diagnóstico y predicción de enfermedades. La Neuroética debería examinar cómo los médicos, jueces y abogados, ejecutivos de compañías aseguradoras y políticos, así como la sociedad en general, tratan con todos estos resultados». En pocas ocasiones la nota de un editor dejó abiertos tantos caminos.

Desde este planteamiento, toda aplicación jurídica de los avances científicos en el conocimiento del funcionamiento cerebral y sus aplicaciones al campo del derecho denotarán de algún modo, construir emplear- la neuroética. He aquí nuestro reto. Recapitulemos. ¿Somos hoy más transparentes? ¿Lo es la manera de cada cual de adoptar las decisiones? ¿Conocemos más acerca de la dinámica formativa de la decisión ética? Pero qué duda cabe que, a estas alturas, estos interrogantes tal vez no puedan responderse sin abordar otros no menos re-

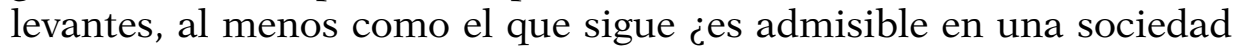
democrática avanzada el empleo de tales medios para alcanzar la verdad, sin considerar su impacto y colisión con los derechos fundamentales?

${ }^{11}$ SÁNCHEZ-MIGALLÓN GRANADOS, S. y GIMÉNEZ AMAYA, J. M. "Neuroética» en FERNÁNDEZ LABASTIDA, F. y MERCADO, J. A. (editores) Philosophica: Enciclopedia filosófica on line: http://www.philosophica.info/archivo/20092008/voces/ neuroetica/Neuroetica.html. 


\section{DESAFÍOS ÉTICO-JURÍDICOS NACIDOS DE LA EVOLUCIÓN NEUROCIENTÍFICA}

En esta clave de diálogo e interrelación entre los campos de la neurociencia y el derecho, centraremos nuestro trabajo en lo que Adina Rooskies ${ }^{12}$ ha identificado como neurociencia de la ética, es decir dejando a un lado las implicaciones éticas de la investigación y la praxis neuromédica, para dirigir nuestros esfuerzos a tareas tales como la redefinición y relectura de nociones jurídicas clásicas tales como el libre albedrío, el autocontrol, la identidad o la intención.

\section{A. Identificación de los desafíos. Autores relevantes}

En 2002 Adina L. Rooskies publica Neuroethics for the New Millenium. Su aportación es esencial por el criterio metodológico aplicado que la literatura posterior ha consagrado y que permite una aproximación sistemática ya clásica. Rooskies distingue entre ética de la neurociencia y neurociencia de la ética. Como anticipamos en este trabajo nos ocuparemos en mayor medida del segundo campo, pero con respecto a la ética de la neurociencia, entiende la autora que encuentra cobijo importantes cuestiones, dentro de dos subgrupos, la ética de la práctica neurocientífica y las implicaciones éticas de la neurociencia. Sin embargo, en la reflexión ético-jurídica que provoca la neurociencia de la ética es, para Rooskies, el terreno propicio donde replantear el alcance de los conceptos éticos tradicionales. En suma, sólo el decurso del tiempo y el desarrollo de la investigación nos permitirán apreciar si las modernas técnicas de neuroimagen nos ofrecen nuevos perfiles de las viejas ideas de libre albedrío, autocontrol, identidad personal o intención. Se trata, una vez más, de comprobar si la realidad del objeto percibido resulta influida por el propio proceso de percepción.

Precisamente de las técnicas de neuroimagen se dedican los esfuerzos de Judy Illes en la obra que escribe junto con Thomas Raffin: "Neuroethics: an Emerging New Discipline in the Study of Brain and Cognition», publicada en la revista especializada Brain and Cognition en el año 2002. El artículo contiene una reflexiva aproximación a las cuestiones neuroéticas desde las posibilidades de visualización de la actividad cerebral. De particular interés neurobiológico resultará la observación tecnológica de los patrones de activación cerebral asocia-

\footnotetext{
${ }^{12}$ ROOSKIES, A. L. "Neuroethics for the new millenium», Neuron, n. ${ }^{\circ}$ 35, 2002, págs. 21-23.
} 
dos a procesos cognitivos o conductuales. En cualquier caso resulta de interés, desde un punto de vista expositivo, las dos líneas de observación neurobiológica analizadas, la del proceso interno de conformación cognitiva y la del proceso externo de generación de la conducta. Al respecto, es destacable la conexión que de este modo se establece entre neurociencia y derecho penal -en la que más tarde abundaremos-, ya que conforme al aserto latino "el pensamiento no delinque», lógicamente el análisis de la responsabilidad distingue la intención -o la ideación- y la acción.

Asimismo, Martha Farah ${ }^{13}$ expone un catálogo de cuestiones que necesariamente habrán de ser abordadas en la oleada de avances neurocientíficos de las próximas décadas, comenzando con el recurso ya esbozado a las técnicas de lectura cerebral. Otra cuestión recurrente en los trabajos de Farah es el análisis de las implicaciones éticas de la tecnología neuroquirúrgica, con especial énfasis en el empleo de la neurofarmacología. Ya en 2002 la autora listaba los principales logros neurocientíficos, y analizando su funcionalidad, trataba de anticipar las resonancias éticas que su generalización provocaría. Así examinaba el tratamiento farmacológico mediante neurotransmisores, para enfermedades como el Alzheimer y el Trastorno de Déficit de Atención e Hiperactividad; asimismo planteaba los efectos que produciría la posibilidad de acordar judicialmente un tratamiento modificador de conductas en personas con comportamiento asocial. En relación a todos ellos efectuaba un análisis ponderado de las contraindicaciones de cada recurso. Así, la posibilidad de acordar judicialmente una terapia para adecuar la conducta de determinadas personas a patrones de normalidad social suscita ya a priori importantes colisiones con derechos fundamentales tales como la integridad corporal y la intimidad, sin perjuicio de que, en determinadas circunstancias y con apropiadas garantías puedan tener cabida en los ordenamientos jurídicos democráticos. Al igual sucede con las técnicas de lectura cerebral, que pese a sus prometedores resultados y su empleo en algunos sistemas judiciales, encuentra importantes colisiones con el derecho a la intimidad personal. Ya entonces otros interrogantes eran tan sólo anunciados, tales como la eficacia y procedencia de las técnicas de TMI aplicadas a la estimulación transcraneal, así como la coadyuvación a un mejor conocimiento del funcionamiento cerebral en la determinación más precisa de la muerte, conforme a los protocolos de Harvard tenidos por óptimos hasta la fecha.

${ }^{13}$ FARAH, M. «Emerging Ethical Issues in Neuroscience», Nature Neuroscience, n. ${ }^{\circ}$ 5, 2002, págs. 1123-1129. 
Para Farah, la neurociencia se nos presenta como la ciencia capaz de revelarnos quiénes somos; esto es, los secretos de nuestra cimentación biológica y de la construcción cerebral de nuestras decisiones sociales, éticas y, por ende, jurídicas ${ }^{14}$. Lo que caracteriza a la nueva ciencia neural es abarcar un ámbito objetivo necesariamente cambiante y sujeto a constante evolución. Por ello, actualizando sus conclusiones de partida, ya en 2005 Martha FARAH ${ }^{15}$ analiza nuevas vertientes nacidas de la problemática neurocientífica. En primer lugar, para desentrañar la incidencia en la privacidad cerebral de los procedimientos de imaginería cerebral, es imprescindible conocer que, junto a las ya citadas tomografía por emisión de positrones (PET) y la resonancia magnética funcional de imágenes (fMRI), actualmente existen técnicas más novedosas y con resultados más efectivos, tales como la magnetoencefalografía (MEG) así como la espectroscopia por infrarrojos (NIRS). Lógicamente, por su mayor grado de incidencia en la práctica jurídica resulta adecuado el examen más exhaustivo de las técnicas que habilitan a la detección de mentiras, en sus correlatos cerebrales. Así, el llamado método «brain fingerprinting» es la base que nos permite hablar del concepto de conocimiento culpable, como aquel diferenciado al que de un evento tendría una persona que simplemente presenció los hechos. Esta técnica de «brainotyping», a semejanza del rastro indeleble de la huella personal como elemento irrepetible y diferenciador de cada persona, de constatarse su coincidencia absoluta con la realidad, abriría importantes puertas a una nueva actividad probatoria. Tales técnicas hasta el momento se han aplicado a la detección de terroristas en controles aeroportuarios, con importantes limitaciones de la intimidad. La cuestión es ¿tales medios son aptos para la comprobación de la culpabilidad de un sospechoso de delitos sexuales, por ejemplo? ¿La huella cerebral detectada mediante el recurso a tales técnicas sería bastante en un estado democrático de derecho para conformar una resolución de condena? Aun en el caso de estar previsto legalmente ¿sería asumible socialmente el riesgo de conocimientos desvirtuados erróneamente detectados? Es decir, ¿qué grado de fiabilidad cabe atribuir por sí solas a estas técnicas sin otra corroboración probatoria que acredite los hechos averiguados? A propósito de esta línea argumental, nos hemos aproximado tangencialmente a lo que llamaríamos una cuestión de neurociencia aplicada al derecho. En efecto, Martha Farah se refiere

\footnotetext{
${ }^{14}$ FARAH, M. Ibidem.

${ }^{15}$ FARAH, M. «Neuroethics: the practical and the philosophical», Trends in Cognitive Science, n. ${ }^{\circ}$ 9, 2005, págs. 34-40.
} 
a los estudios de Elizabeth Loftus ${ }^{16}$ en el campo de la memoria, como el más certero enfoque científico de la huella cerebral de la memoria, lo que abre un vasto campo de colaboración en materia probatoria, por las vías de las declaraciones testificales y la reconstrucción de hechos. Cumplido el propósito expositivo perseguido, en otro apartado de este trabajo nos centraremos en las consecuencias prácticas que pueden ofrecer los métodos neurales de aproximación a la memoria.

En 2007, Farah publica un tercer trabajo con vocación generalista $^{17}$ centrado en los desafíos suscitados por la neurociencia cognitiva. Así, las cuestiones neurocientíficas de mayor interés de los últimos años guardan relación con dos fenómenos técnicos: la creciente capacidad de monitorizar la función cerebral en seres humanos con resolución suficiente como para captar fluctuaciones de actividad significativas que, combinado en su justa proporción con las mejoras en el análisis -interpretación- de datos abre una vía de acción decisiva en la observación de la personalidad humana, incluso en su tratamiento; y de otro lado, la posibilidad técnica de alterar la función cerebral humana a través de mecanismos de selección químicos o anatómicos capaces de inducir cambios psicológicos específicos, que abriría la posibilidad de empleo de las técnicas exploradas más allá de los límites del terreno clínico, con potencial eficacia a la mejora en cualquier ser humano de su función cerebral normalizada. Aunque en otros epígrafes de este trabajo dedicaremos mayor atención, baste señalar ahora que, junto a los procedimientos farmacológicos, se abre la posibilidad de activación cerebral profunda mediante la estimulación magnética transcraneal y la directa mediante corrientes.

Jonathan Moreno es un referente en materia de defensa en su relación con la neurociencia. Ya en su primer artículo ${ }^{18}$ sobre neuroética publicado en 2003, planteaba una interesante analogía entre la explosión de la genética contemporánea - a finales de siglo xx- y la eclosión de las ciencias cerebrales, en los primeros años del siglo XXI, que ya hacían presagiar que se abría una nueva etapa de la historia humana, la era del cerebro. Moreno contextualiza su análisis en el marco del pensamiento de Patricia Churchland, de manera que previsiblemente la respuesta al gran interrogante de la libertad humana, vendrá en esta nueva etapa por la necesaria interrelación entre Neu-

${ }^{16}$ LOFTUS, E. «Our Changeable memories: Legal and Practical Implications» Nature Reviews / Neuroscience, Vol. 4 (marzo), 2003, págs. 231-233.

${ }^{17}$ FARAH, M. «Social, Legal and Ethical Implications of Cognitive Neuroscience: «Neuroethics» for Short», Journal of Cognitive Neuroscience, n. ${ }^{\circ} 19,2007,363-364$.

${ }_{18}$ MORENO, J. D. "Neuroethics: An Agenda for Neuroscience and Society», Nature Reviews in Neuroscience n. ${ }^{\circ}$ 4, 2003, págs. 149-153. 
rociencia y Filosofía. Y es que, en el fondo, los grandes conceptos que replantea con nueva perspectiva la neurobiología se identifican con cuestiones humanas de todo tiempo ${ }^{19}$. Pero tal vez el aspecto más conocido, por específico, de la obra de Moreno se residencia en la concreta aplicación de la nueva disciplina neurocientífica a la esfera militar. Así, si Gazzaniga apadrinará el concepto de cerebro ético, la gran aportación de Moreno es la profundización y referencia a la noción de guerra mental -o cerebral- ${ }^{20}$. De este modo, la investigación en defensa pasa a tener un matiz distintivo en su nueva estrategia de comprensión desde su clave neural. Hasta tal punto que, como veremos, Neil Levy llegará a cuestionarse dónde se encuentra en realidad la mente.

\section{B. La inevitable conexión Neurociencias-Derecho Penal}

Aunque los artículos y monografías publicados en los últimos años son ingentes al respecto, el gran interrogante que se plantea es el de qué relevancia tendrán las aportaciones de la Neurociencia en el estadio actual y en el desarrollo venidero del Derecho Penal. Es decir, si los innegables aportes derivados de los descubrimientos en la ciencia y técnica neural de las últimas décadas determinarán un cambio radical en la concepción y planteamiento de esta rama del derecho o si por el contrario serán percibidos por los aplicadores y pensadores del ordenamiento jurídico penal tan sólo como nuevas descripciones de una realidad secular. Claro está que entre ambos extremos pueden encontrar acomodo posiciones intermedias como es la de otorgar cierta energía revitalizante y transformadora respecto de algunas de las más importantes nociones de la responsabilidad y el castigo, sin que ello entrañe necesariamente una revolución radical. Veamos.

Entre los autores que más atención han dedicado a la cuestión, merece ser destacada la clarividente visión de Bernardo Sánchez, Feijoo. En un importante trabajo ${ }^{21}$ expone sus principales ideas, con profusa cita de la doctrina alemana. Curiosamente Sánchez Feijoo regresa ilustrativamente a la historia de un auténtico mito de la neurociencia en su dimensión aplicativa a las ciencias sociales, Phineas

${ }^{19}$ MORSE, S. J. «New Neuroscience, Old Problems: Legal Implications of the Brain Science», Cerebrum n. . 6, 2004, págs. 81-90.

${ }^{20}$ MORENO, J. D. Mind Wars. Brain Research and National Defense, The Dana Press, New York, 2006.

${ }^{21}$ FEIJOO SÁNCHEZ, B. «Derecho Penal y Neurociencias. ¿Una relación tormentosa?»InDret n. ${ }^{\circ}$ 2/11, págs. 39-48. 
Gage. Muy recomendable al respecto es la obra de Antonio Damasio El Error de Descartes.

En el sentido expuesto, la principal influencia que pueden ejercer las neurociencias se apreciará en la percepción acerca de la capacidad de culpabilidad, de manera que los logros en neurotecnología -y neuroimagen- desembocarán previsiblemente en una ampliación de supuestos de inimputabilidad y semiimputabilidad. La cuestión por resolver estriba en qué casos es normativamente razonable renunciar a las penas por tratamiento. Desde este enfoque, por lo tanto, el artículo 20.1. ${ }^{\circ}$ C. Penal, representa una puerta abierta a la colaboración de las neurociencias y el derecho. Asimismo apunta Sánchez Feijoo entre los aspectos problemáticos de la cada vez más ineludible conexión que, a día de hoy, los diagnósticos individualizados mediante neuroimagen sólo sirven para constatar diagnósticos previamente realizados con métodos tradicionales. De manera que, ante tan parcial eficacia real, resta por delante un arduo proceso de elaboración social, lo que no es una novedad, sino más bien un vínculo de comunicabilidad con lo analizado para el derecho aplicado en general. Así, podemos afirmar que el Derecho Penal de hoy aguarda la mayoría de edad de las Neurociencias, al menos en cuanto a la consagración de los procedimientos neurotecnológicos -particularmente los de imaginaría cerebral- como métodos autónomos con potencial valor probatorio pleno.

De este modo la Neurociencia coquetea con el Derecho Penal para convertirse en la linterna que alumbre nuevas realidades de ausencia o disminución de la capacidad. Si el fundamento de la condena radica en la ignorancia o no constatación de explicaciones alternativas a la responsabilidad -la disposición jurídica del individuo-, el efectivo avance de las neurociencia sea efectivo contribuirá a diluir nuestras ignorancias, lo que tendrá efecto en la práctica forense de los próximos años.

Estas conclusiones son fruto elaborado sobre la base del estadio actual de las investigaciones sobre el funcionamiento del cerebro, entendido como el órgano que crea por emergencia, mediante procesos neuronales la consciencia y los procesos psíquicos. Estos procesos psíquicos crean por emergencia un orden social. Y este orden social -cuyas raíces cerebrales rastrea la Neurociencia- es la realidad que el Derecho normativiza y, mediatamente, normaliza

Por todo ello, en el fondo último del debate juristas/neurocientíficos, siempre nos topamos con un problema categorial: los juristas, reflexionan a largo plazo; los procesos neuronales tienen lugar a corto. En suma, aunque la ciencia pueda explicar pormenorizadamente 
cada paso del proceso cerebral humano, el ciudadano es un ser libre, desde una perspectiva intersubjetiva. De este modo, los neurotransmisores y los preceptos normativos no sólo tienen ritmos diferentes, sino que asimismo hablan lenguajes diferentes. Por tanto la exigencia de una colaboración bidireccional entre Derecho y Neurociencia pasará por hallar las claves de una mutua comprensión de los idiomas en que cada cual se expresa. La relevancia de los avances neurales exige un diálogo fértil en que cada disciplina asimile e incorpore criterios y nociones ajenos. Por ello, a la gran cuestión que sobrevuela el debate del determinismo, la conexión Derecho Penal-Neurociencia nos ofrece la respuesta de que no estamos programados, sino neurológicamente determinados. El determinismo actual implica que los comportamientos humanos van mostrándose paso a paso, entrecruzándose factores diversos. De ahí que las neurociencias sólo modificarán radicalmente la noción jurídico-penal de culpabilidad si logran previamente modificar radicalmente la propia configuración normativa de la sociedad y sus principios. Es decir, la efectividad del enorme potencial transformador de la Neurociencia respecto del Derecho Penal dependerá de que previamente transforme la sociedad.

Por ello concluye que las neurociencias perfilarán una nueva autocomprensión del ser humano, sin que sea imprescindible una revolución de las bases de la organización social. Por ello la ciencia del derecho penal no debería perder la cara a la neurociencia, contemplando con asombro científico cada espectacular avance sobre el conocimiento del funcionamiento cerebral. Una vez más, el secreto es la clave neural.

Conectada doctrinalmente con las posiciones de Sánchez Feijoo, Mercedes Pérez Manzano suscribe un artículo ${ }^{22}$ con aportaciones más apegadas a la práctica forense, relacionando, con admirable sentido pedagógico el impacto de la revolución neurocientífica con el propio funcionamiento del sistema jurídico-penal. La autora sitúa el momento inicial de esta consagración en la publicación en el año 2004 en Alemania, bajo el nombre «Das Manifest», de un manifiesto sobre las repercusiones de los avances neurocientíficos en los diferentes sectores del saber. En el derecho penal, en particular, se planteaba su incidencia en el fundamento de la responsabilidad penal y de la sanción penal. La cuestión es si refutarán las neurociencias la tesis de que el ser humano actúa de forma libre y voluntaria. Es decir, la reformulación del viejo interrogante determinista, ahora en dimensión neural.

${ }^{22}$ PÉREZ MANZANO, M. «Fundamento y Fines del Derecho Penal. Una revisión a la luz de las aportaciones de la Neurociencia», InDret n. ${ }^{\circ}$ 2/11, págs. 2-31.

(C) UNED. Revista de Derecho UNED, núm. 17, 2015 
En su opinión, las aportaciones de la neurociencia son imprescindibles para entender los mecanismos psicofísicos que posibilitan la propia eficacia regulativa de conductas del Derecho. La explicación del funcionamiento de los procesos neurológicos es fundamental para entender el funcionamiento del Derecho Penal como sistema llamado a incidir en la conducta de los ciudadanos a través de la sanción. De este modo, la necesidad de disponer de la «clave neurológica» se explica en su idoneidad para comprender el funcionamiento del derecho penal, para mejorar sus instrumentos y para fundamentar racionalmente sus elementos conceptuales y fines. Por lo tanto, la propuesta de Pérez Manzano es sumamente ambiciosa y anticipa que estudios posteriores abundarán en estas líneas de investigación. En realidad, del planteamiento asumido se atisban tres grandes dimensiones para el análisis: mecánica, instrumental y teleológica.

Así pues, solventadas las primeras cuestiones de partida, qué duda cabe que los hallazgos en el campo neurocientífico afectarán a uno de los núcleos duros de la ciencia penal. Es el caso de la sacrosanta noción de la imputabilidad. A la cuestión de si los conocimientos neurales determinan algún cambio en el modo de percibir la imputabilidad, la respuesta ha de ser hoy decididamente afirmativa. En realidad, el enfoque neural nos ofrece elementos para entender que el juicio de imputabilidad neurológicamente implica conexiones entre niveles de funcionamiento sub-personal (cerebral) y personal (realización de la conducta humana). De manera que médicamente puede ser entendido como un enlace entre dos planos o niveles de la realidad biológica.

En este sentido, la neurociencia cognitiva ha aportado datos sobre dos clases de sistemas cognitivos y procesos neuronales: objetos y procesos físicos y conductas intencionales de agentes. En este contexto, hallazgos neurocientíficos respaldarían que la tendencia psíquica a reaccionar con una sanción justa proporcionada sería producto de viejos mecanismos filogenéticos que acontecen en el cerebro -the neural basis of economics decision-making in the ultimtum game- y que estos mecanismos serían eficientes para la estabilidad social-neurobiology of punishment. Así se abre un interesante ámbito de relación futura entre la neurobiología del castigo y la ciencia del Derecho Penal, cuya recíproca influencia incidirá con seguridad en la determinación conceptual, así como en la redefinición de medios y fines.

En lógica consecuencia, la incidencia de esta apreciada inclinación a criterios neurocientíficos por parte de derecho penal conllevará necesariamente el tránsito de la pena a la medida preventivo-especial y, dentro de ésta, al protagonismo del tratamiento terapéutico neuro- 
lógico, como forma sustitutiva de la sanción penal pura. Una visión en perspectiva histórica nos recordará sin duda los posicionamientos constituyentes de la Escuela Positiva italiana, entre cuyos autores más notables, se propusieron las nociones de peligrosidad social y tratamiento, como contrapuestas a culpabilidad y castigo. Las consecuencias de esta concepción global propuesta de un derecho penal de medidas -se entiende, con vocación neurocientífica-, basado en la peligrosidad criminal y orientado a la prevención especial, harían posible que la tarea de legislador y aplicador del derecho consistiera en fijar el "quántum» de la medida partiendo del parámetro de la peligrosidad del sujeto, debiendo dejarla indeterminada para ser precisada posteriormente y en función del éxito del tratamiento. Otro ejemplo más de la necesidad de reorientar el punto de mira del delito al sujeto delincuente.

Por esta vía alcanzamos uno de los aspectos donde el aporte neurológico cobra más sentido. Si la pena, respecto a grupos de población determinados, habrá de ser sustituida por la medida de seguridad y uno de los elementos básicos de ésta viene constituido por el tratamiento, hemos de plantearnos en qué medida la nueva comprensión neurobiológica del ser humano incide en la determinación del tratamiento como medida de respuesta a la peligrosidad. El tratamiento neurológico -en tanto terapia social emancipadora- exigirá ponderar la magnitud de la injerencias en el cerebro en cada caso concreto, en atención a la peculiar significación del cerebro como fuente de nuestra consciencia y del sentimiento del yo; como constitutivo de nuestra subjetividad; $y$ presupuesto fisiológico de nuestra identidad. En este punto, si el cerebro no es un órgano más, sino que se le atribuye un elemento constitutivo y fundacional de la subjetividad y personalidad, toda intervención directa o indirecta habrá de ser examinada cuidando los detalles, por su inmediata conexión con derechos humanos como la intimidad personal. No obstante, no es necesario acudir a situaciones experimentales o "de laboratorio». Sin ir más lejos, en nuestro ordenamiento penal vigente, el tratamiento tiene cabida y puede revestir el contenido de la privación de libertad -como pena o medida de seguridad-; puede sustituir a la pena; o puede finalmente integrar los condicionantes de la suspensión. Su potencial de incidencia en los derechos fundamentales es enorme, de ahí que la protección penal de la norma opera como la otra cara de la moneda de la efectividad de los derechos fundamentales.

Otro punto de enorme interés se residencia en si la Neurociencia ofrece datos sobre la correlación entre déficits neurológicos y perpetración de delitos, pues ello abriría un razonable desafío en orden a ex- 
plorar las posibilidades del tratamiento neurológico para remover las causas neurológicas que influyen en la comisión. Esta posibilidad cierta hoy abre la puerta a mecanismos como castración química aplicada a delincuentes sexuales, aunque asimismo lógicamente a otras opciones menos radicales, cual sería el caso a título de ejemplo, el recurso a métodos farmacológicos en relación a delitos asociados a la habituación al consumo de alcohol o drogas, o la prescripción de ansiolíticos a los internos en régimen penitenciario para mejor afrontar la pacífica convivencia en los centros. En este sentido Greely ${ }^{23}$ da un paso más y plantea posibilidades de intervención quirúrgica claramente invasivas, como sería el caso de extirpación de un tumor cerebral que afectase al funcionamiento de ciertas zonas cerebrales. Como vemos, la casuística es inabarcable. En suma, desde un punto de vista médico-neurológico, se trata de abordar las ventajas que pueden aportar los estudios neurológicos avanzados; desde un punto de vista ético-jurídico se plantea en qué medida esta posibilidad científica puede colisionar con los Derechos Fundamentales de la persona. Desde este planteamiento, cabe distinguir en el análisis dos situaciones: el tratamiento neurológico puede concebirse como contenido efectivo de una pena o como parte de una medida de seguridad. Lo trascendente es que esta opción aparentemente sólo con importancia a nivel del ordenamiento jurídico penal, va a implicar relevantes consecuencias en el plano de los Derechos Humanos, por el cauce de su carácter o no voluntario. De ser admisible como pena sería requisito su carácter voluntario, sobre la base de un juicio previo de culpabilidad. En esta lógica de partida, podría plantear problemas su anudamiento a beneficios para el penado, en tanto que tal conexión puede hacer duda de la espontaneidad y sinceridad del carácter voluntario. Esta situación no es una hipótesis sino que tendría cabida en el C. Penal español vigente (art. 87.1 C P), para los supuestos de condenados que se someten a tratamiento de deshabituación de drogas o alcohol, en los como medio de obtener la suspensión de su condena a prisión. De ser admitido como medida aplicable a los inimputables, habría de ser impuesto por la institución competente, sobre la base del reconocimiento de su propia incapacidad para comprender el significado jurídico de sus actos y aceptar la respuesta. En relación a estos supuestos, la problemática más importante que se suscita desde el punto de vista del respeto al derecho fundamental a la intimidad es el de legitimación de la autoridad o institución pública cuando tales terapias sean susceptibles de generar efectos permanentes.

${ }^{23}$ GREELY, H. T. «Neuroscience and Criminal Justice: Not Responsibility but Treatment», Kansas Law Review n. ${ }^{\circ}$ 56, 2008, págs. 1103 y ss. 
En particular, atendida la especificidad del cerebro como fuente de consciencia y personalidad, en un análisis ético-jurídico riguroso, se impone la necesidad de dotar de un estatus jurídico singularizado a los supuestos en que se recurra al tratamiento neurológico, sin descartar que prospere la prohibición de las intervenciones profundas en el cerebro de carácter permanente e irreversible, dado que, frente al postura ancladas en alguna suerte de paternalismo estatal, se impone el respeto a los derechos fundamentales y principios constitucionales en juego, a saber, el libre desarrollo de la personalidad y la dignidad humana (art. 10.1 CE) y los derechos fundamentales a la identidad personal y a la identidad física y moral (art. $15 \mathrm{CE}$ ).

En un nuevo análisis profundo de la cuestión de las relaciones entre Neurociencias y Derecho Penal, Eduardo Demetrio Crespo ${ }^{24}$ ha ahondado en el impacto de esta conexión en las nociones troncales de libertad, voluntad, investigación cerebral y responsabilidad. La neurobiología tiene algo que decir en el viejo debate entre determinismo e indeterminismo. Si el quid radica en si es posible la libertad humana, cualquiera que sea la respuesta podrá ser neurobiológicamente «observada» desde los instrumentos técnicos y experimentales nacidos al amparo de la ciencias neurales contemporáneas. Hoy es posible que las neurociencias induzcan una definitiva transformación desde los hallazgos científicos relativos al comportamiento humano. Este potencial caudal transformante derramará su energía en una labor insólita de inculturización, esto es, la neurociencia no arramblará con las nociones clásicas de la dogmática jurídico-penal -como la responsabilidad y el libre albedrío-, pero representan hoy evidentemente el aldabonazo a una nueva cultura social y del pensamiento, a la que necesariamente habrá de conectarse el derecho penal. Así, Demetrio Crespo propone parcelas concretas a que debería alcanzar la nueva cultura neural, entre las que imperiosamente estarán las causas de inimputabilidad y ello en tanto que la culpabilidad no es sino una construcción social, de ahí que haya de ser definida conforme a los postulados sociales necesariamente cambiantes al albur de los tiempos.

De particular interés son algunas de las conclusiones personales que alcanza Eduardo Demetrio como lo es considerar que el problema determinista en realidad esconde lo que denomina un «juego de lenguaje»-si somos o no libres dependerá del concepto de libertad que barajemos-, en contraste a la postura meridianamente indeterminista de Vives Antón. Precisamente el Derecho Penal, en cuanto cien-

${ }^{24}$ DEMETRIO CRESPO, E. «Libertad de Voluntad, Investigación sobre el Cerebro y Responsabilidad Penal: aproximación a los fundamentos del moderno debate entre Neurociencias y Derecho Penal», InDret, n. ${ }^{\circ}$ 2/2011.

(C) UNED. Revista de Derecho UNED, núm. 17, 2015 
cia humana, no debería permanecer ajeno a los nuevos «juegos de lenguaje» que comienzan a aportar en imparable corriente las Neurociencias. Estos juegos en realidad serán las nuevas lentes desde las que observar con la mayor precisión el nuevo universo conceptual. Además de un acercamiento imprescindible, tal vez represente una excelente estrategia para superar el vicio normativista de la dogmática penal. Así las cosas, cabe preguntarse en último lugar si el clásico problema categorial entre unas y otras ciencias se inclina más hacia el defecto de las Neurociencias por interferir en la definición del sistema penal de responsabilidad, o bien hacia el Derecho Penal por ignorar a sabiendas la base empírica mínima.

\section{LA NEUROÉTICA EN RELACIÓN CON LOS DERECHOS HUMANOS}

Aprovecharemos en este punto la ya clásica diferenciación patrocinada por Adina Rooskies, como ética de la neurociencia y como neurociencia de la ética.

Desde la perspectiva de ética de la neurociencia, la neuroética nace con vocación de definir los límites a los avances de la Neurociencia, a modo de diques infranqueables frente a las opciones científicas que no resisten el debate ético. En este sentido, la Neuroética cumple la importante misión de definir el contenido de las bases materiales de esta nueva rama de la ética. En tal función, operará en calidad de bioética aplicada, sentando criterios para decidir lo válido y lo inválido siempre desde el baremo que representan los derechos fundamentales. En este marco, la perspectiva iusfundamental se adivina como estrategia idónea para contrarrestar el espíritu innovador de las modernas técnicas de neuroimagen, en una reedición actualizada del debate entre bioética y bioderecho. La dinámica Neuroética y Derechos Humanos acentuará la función controladora de ésta respecto de los excesos tecnológicos, presentando los Derechos Humanos una función integrativa del contenido y límites a la ciencia.

Desde la de neurociencia de la ética, la Neuroética cobra su auténtica dimensión diferencial y de rama científica de nuevo cuño. Desde este enfoque es posible analizar cada etapa del proceso neuronal de toma de decisiones. Habrá de plantearse la compatibilidad de la resonancia magnética funcional o de la electroencefalografía cerebral o la tomografía de positrones con derechos fundamentales tales como la intimidad personal o la integridad corporal, así como por valores superiores constitucionales como la dignidad humana (art. 10.2 CE). De 
particular relevancia serán las consecuencias derivadas del empleo de las técnicas de imaginería cerebral, entendidas en la doctrina del Tribunal Constitucional como injerencias o intromisiones corporales. ¿Presentan éstas alguna especialidad en cuanto a su régimen jurídico en tanto que la inmisión es de tipo cerebral? De la amplitud otorgada a estas inmisiones en determinados supuestos dependerán algunos de los medios de prueba llamados a ser decisivos en el futuro, como es el caso de la huella de memoria o el registro de potenciales evocados, que habrían de ser considerados en el proyecto de reforma de la Ley de Enjuiciamiento Criminal.

Esta propuesta esquemática relacional entre la neuroética y los derechos humanos encuentra una aplicación concreta ya esbozada ${ }^{25}$. Se trata de la opción del recurso al denominado tratamiento neurológico, donde las exigencias de comprobación científica de la efectividad de los métodos habrán de ser proporcionales a la magnitud de los daños potenciales; los requisitos y autorizaciones habrán de ser individualizados en relación a cada fármaco o procedimiento en función del uso y efectos pretendidos; y el empleo de las terapias neurológicas en delincuentes deberá sujetarse a controles particularmente rigurosos. Por tanto, y frente a percepciones tradicionalmente asumidas, la diferencia entre las terapias psicológicas y educativas tradicionales y los tratamientos neurológicos radicará en el empleo en las primeras de un método indirecto y en que los segundos pueden ser más aflictivos e incidir en la identidad personal. En este sentido no habrá que atender tanto a la velocidad ni al carácter directo cuanto a la producción de sus efectos -que no los convierte en ilegítimos-, cuanto a si de los mismos se desprende un efecto negativo o afectante de la identidad personal. Se impone, en consecuencia, la decisiva tarea de la comunidad científica y de las instituciones jurídicas de contrastar debidamente la validez de las terapias individuales como respuesta al delito, incluso en el caso de los tratamientos voluntarios. A priori, el parámetro del artículo 10.1 de la CE parece colisionar con tratamientos neurológicos que afecten de forma permanente al núcleo de la personalidad. Particularmente atendida la especificidad del cerebro como fuente de consciencia y personalidad, procediendo probablemente la prohibición de las intervenciones profundas en el cerebro de carácter permanente e irreversible, dejando a un lado en este caso por sus propias características posiciones más proclives al paternalismo estatal o institucional. En tanto núcleo y fuente de la personalidad y consciencia humanas la ética exige el repudio de tales actuaciones cuando revistan carácter permanente, en tanto desnaturalizarían el

${ }^{25}$ PÉREZ MANZANO, M. Op.cit., (2-31). 
núcleo mismo de la subjetividad e irrepetibilidad del ser humano, de manera que por afectar gravemente a su dignidad colisionarían de modo irresoluble con los derechos fundamentales consagrados, constitucionalmente y en las declaraciones de carácter internacional.

\section{APORTACIÓN DE LA NEUROÉTICA A LA CONSTRUCCIÓN DE UNA ÉTICA UNIVERSAL. DEL «ES» CEREBRAL AL «DEBE» MORAL}

Son muchos los autores que se han ocupado de tamaña cuestión. La clave radica en que si la identificación de un código ético universalmente aplicable permitiría prescindir de abstracciones formalistas, de modo que sin duda su huella primigenia estaría sellada en el cerebro humano y de ahí que los juicios morales se hallaran en realidad ínsitos en cada persona, que sólo tendría que aplicarlos cuando obra. Sin embargo, con toda probabilidad ha sido Adela Cortina quien se ha ocupado de una manera más generalista, directa, pedagógica y amena de la cuestión. Primero con un artículo ${ }^{26}$, y posteriormente en una espléndida monografía ${ }^{27}$ en que desarrolla algunas de las ideas esbozadas y ensancha el campo de acción de la nueva ciencia, al tiempo que profundiza en su repercusión.

Coincidimos con Cortina en que la noción de neurociencia de la ética nos abre un inmenso panorama que permite plantearse si la neuroética representa una verdadera revolución. Si la sala de las neurociencias custodia la llave del fundamento cerebral de una ética normativa. Es la posición que defienden nítidamente autores como Gazzaniga quien, con su definición de neuroética en tanto propuesta de una filosofía de vida con fundamento cerebral, en realidad ya ha encontrado el sustituto de las construcciones ideológicas y religiosas que acabaría con sus intentos monopolizadores de la idea de una clave ética universal. Cortina subraya la endeble consistencia de algunas de las nociones que cimentan con más solidez el consenso de los neurocientíficos, de modo que ante la efectiva unanimidad acerca de que la intuición de lo que es correcto o incorrecto en el caso concreto es base de nuestros juicios morales, cuestiona qué entender en una clave

${ }^{26}$ CORTINA, A. "Neuroética: ¿las bases cerebrales de una ética universal con relevancia política?», ISEGORÍA. Revista de Filosofía Moral y Política n. ${ }^{\circ} 42,2010$, págs. 129-148.

${ }^{27}$ CORTINA, A. Neuroética y Neuropolítica: Sugerencias para la Educación Moral, Ed. Tecnos. Madrid, 2011 págs. 72-76. 
científica por intuición. Si hablamos de instinto, sentido o tal vez competencia.

En esta línea, los estudios de Greene ${ }^{28}$ revelan que la neurociencia tiene mucho que decir a lo hora de aclarar la diferente reacción de los sujetos que se enfrentan a dilemas subjetivos a cuando se topan con dilemas impersonales. En estos meditados juicios radica uno de aspectos fuertes de la mecánica discursiva neuroética, en tanto que es estadísticamente un hecho constatado cómo el individuo reacciona de manera diferenciada ante estos dilemas en función de que éstos susciten conflictos éticos personales o impersonales. ¿Qué tiene que aportar la neurociencia en la resolución de tales dilemas? La aplicación técnica experimental de Greene consistió en el escaneo de la actividad cerebral en sujetos mientras leen dilemas éticos. Hay que tener en cuenta como punto de partida que Mora $^{29}$ viene a definir la lectura cerebral como "capacidad técnica y (...) conocimientos científicos para llegar a conocer lo que piensa una persona sin que lo manifieste a partir de los registros de actividad cerebral». Es decir, las modernas técnicas de neuroimagen nos permiten saber sin hablar. Comunicar sin expresar. Es obvio que un punto de inflexión tan relevante comportará no pocas resonancias y cuestiones ético-jurídicas por resolver. Gazzaniga ${ }^{30}$ incluye entre el grupo de nuevas tecnologías que comportan procedimientos para leer mentes, además de la resonancia magnética funcional (fMRI), al registro de la actividad eléctrica del cerebro y los sensores de calor, entre otros. En este contexto, los estudios de Greene evidenciaron que en los conflictos morales personales, las imágenes cerebrales revelaban una intensa actividad en zonas básicas en el procesamiento de las emociones, desde el lóbulo frontal al sistema límbico. De otro lado, se comprobó cómo cuando el sujeto formulaba un juicio a contra corriente -como cuando se concluye que es lícito ocasionar perjuicio a una persona en determinadas circunstancias- era evidente una activación mayor del córtex prefrontal dorsolateral, zona que desempeña un papel decisivo en la planificación y el razonamiento.

Cortina asocia la mayor actividad apreciada en las áreas cerebrales responsables de la emoción y la cognición social a que la relación

${ }^{28}$ GREENE, J. D. (coord.) «From Neural «Is» To Moral «Ought». What Are The Moral Implications of Neuroscientific Psychology?», GLANNON, W. (Ed.) Defining Right and Wrong in Brain Science, Nature Reviews Neuroscience, Vol. 4, 2003, New York, Ed. DANA Press, págs. 847-850.

${ }^{29}$ MORA, F. «El Cerebro humano: desafíos para el siglo XXI», Eidon n. ${ }^{\circ} 33,2010$, págs. 48-51.

${ }^{30}$ GAZZANIGA, M. S. El cerebro ético, Ed. Paidós, Barcelona, 2006. 
de cercanía pone en funcionamiento códigos morales-emocionales profundos de supervivencia, lo que no sucede ante dilemas impersonales o personales con menor capacidad de implicación por proximidad. En ello Cortina residencia la función adaptativa que comporta la capacidad universalmente extendida de diferenciar entre el bien y el mal. Claro que más adelante descubriremos cómo no toda la vida ética de la persona se agota en los dilemas de laboratorio. Este cordón adaptativo nos permite coser dos mundos, el del «ser» empírico y el del «deber ser», en la medida que nos faculta para dotarnos de un código ético garante de la supervivencia de la especie humana. La neuroética se carga, por tanto, de fundados argumentos para mostrarnos que las normas morales son en realidad reglas adaptativas.

La siguiente cuestión que nos surge tras interpretar que las normas morales cumplen una tarea adaptativa es identificar de qué normas hablamos; ¿qué normas con contenido podemos extraer del conocimiento de las bases cerebrales de la conducta humana? Se trata de dilucidar si existen códigos morales grabados a fuego en el cerebro, como apunta Mora, o más bien habremos de concluir que del «es» de la supervivencia humana no se desprende inexorablemente el «debe» moral consecuente. Si no, habremos de conmutar la regla de oro «haz a los demás lo que quieres que te hagan» por la de «obra con los demás en función a su proximidad genética contigo».

Finalmente, Adela Cortina acierta a identificar serias deficiencias en las expectativas generadas en torno a la neuroética, entendida ambiciosamente como el estudio de los circuitos cerebrales (y su actividad) que propician el ser ético. Ante la posibilidad de tan altos logros, la neuroética se topa con el escollo ya enunciado referido a la ambigüedad latente en la indefinición del punto de partida -intuiciones, instintos, sentido, competencia o juicios meditados-. Más importante aún es la objeción referida a la mecánica y dimensión misma de la vida moral que, como sostiene Conill $^{31}$, no consiste en enfrentarse a controvertidos dilemas, sino en proyectar una vida buena con la riqueza experiencia consiguiente y no reconducible al plano de los juegos lógicos.

Asimismo, desde el plano sustantivo-jurídico, puede existir contradicción entre los mandatos éticos inscritos en los lóbulos cerebrales y los derechos fundamentales positivizados en las declaraciones internaciones, como la de Derechos Humanos de 1948. Y es que la cuestión última en derecho es la de la legitimación que, en el marco de un Estado Democrático de Derecho sólo puede emanar de la dignidad de

${ }^{31}$ CONILL, J. Ética Hermenéutica, Ed. Tecnos, Madrid, 2006. 
la persona y los derechos inviolables inherentes (artículo 10.2 Constitución Española).

Por ello, concluye Cortina que son tres las posturas que cabe adoptar en la exégesis y el diagnóstico de la relación neurociencias y ciencias sociales en general y derecho en particular.

- La primera sería defendida por autores como Gazzaniga o Mora que enarbolan hoy la posibilidad cierta de formular una ética universal sobre bases cerebrales, sin aclarar qué normas integrarían tal imperativo adaptativo.

- La segunda postura pasaría por defender la posibilidad de comprender la estructura por la que las personas formulan juicios morales, al modo de una «gramática moral» inscrita en el cerebro de cada cual, como defienden Hauser o Cela.

- Finalmente un tercer grupo de autores defiende, como Levy, que las normas éticas tenidas por válidas cambian en la propia función adaptativa histórica del ser humano.

\section{CONCLUSIONES}

1. Los avances neurocientíficos hacen posible hablar hoy de un mundo neurocéntrico, en afortunada expresión de Steven Rose, donde la clave neuroética es imprescindible para valorar la eticidad de los avances neurotecnológicos.

2. Las aportaciones de la Neurociencia son imprescindibles para entender los mecanismos psicofísicos que posibilitan la propia eficacia regulativa de conductas del Derecho. La explicación del funcionamiento de los procesos neurológicos es fundamental para entender el funcionamiento del Derecho Penal como sistema llamado a incidir en la conducta de los ciudadanos a través de la sanción. De este modo, la necesidad de disponer de la «clave neurológica» se explica en su idoneidad para comprender el funcionamiento del derecho penal, para mejorar sus instrumentos y para fundamentar racionalmente sus elementos conceptuales y fines.

3. La Neurociencia ofrece argumentos a favor de que el tratamiento neurológico rehabilitador pueda ser el contenido idóneo de la reacción penal -frente al acto ilícito-, entroncando con la perspectiva de la prevención especial.

4. La función controladora de la Neuroética es hoy ineludible, en cuanto que para la aprobación y legitimidad de posibles terapias neu-

(C) UNED. Revista de Derecho UNED, núm. 17, 2015 
rológicas se antoja necesario un singular control en una doble dimensión: respecto a la suficiencia de los conocimientos científicos y en el proceso de autorización.

5. La base cerebral del juicio moral se desdobla en su proyección neurobiológica: el juicio moral ante un dilema personal pone en marcha las áreas cerebrales vinculadas a las emociones; el dilema impersonal activa las zonas neurales asociadas al razonamiento

6. Frente a posiciones que comienzan a atisbar el sustrato cerebral de una ética universal -e intemporal-al modo kantiano, no puede soslayarse -como afirma Cortina-un escollo ineludible en que tales códigos inscritos en el cerebro humano -y por tanto, universales y no susceptibles de condicionamiento cultural o temporal- puede plantear disonancias notables con valores y derechos como los consagrados en la Declaración de Derechos Humanos de las Naciones Unidas de 1948.

7. No es su base cerebral la característica definitoria de la nueva ética resultante de las aportaciones más recientes de la Neurociencia, sino que en ésta se encuentra el germen de una ética universal con fundamento cerebral.

8. En el fondo último del debate juristas/neurocientíficos, siempre nos topamos con un problema categorial.: los juristas, reflexionan a largo plazo; los procesos neuronales tienen lugar a corto. En suma, aunque la ciencia pueda explicar pormenorizadamente cada paso del proceso cerebral humano, el ciudadano es un ser libre, desde una perspectiva intersubjetiva.

9. El recurso al tratamiento neurológico pasa por ponderar la magnitud de las injerencias en el cerebro en cada caso, en atención a la peculiar significación del cerebro como fuente de nuestra consciencia $y$ del sentimiento del yo; como constitutivo de nuestra subjetividad; $y$ presupuesto fisiológico de nuestra identidad. En consecuencia, plantea una evidente colisión con los derechos fundamentales a la integridad corporal y a la intimidad personal, que habrán de ser valorados en su caso conforme a la doctrina del TC y TEDH.

10. El papel «mesiánico» atribuido a los avances y aplicaciones neurocientíficas es homologable en el terreno de la ética, al papel desempeñado por el derecho natural, con base teológica y racionalista. Por lo tanto, conferir a las neurociencias autoridad para determinar el contenido material de los derechos subjetivos entraña el riesgo histórico de maximizar los contenidos y garantías jurídicas por el camino de una nueva deidad, en este caso científica. El derecho no debe relacionarse con la neurociencia en una posición de subordinado a autoridad. 
11. En la dinámica relacional necesaria entre neurociencia y derechos humanos, la reflexión neuroética tiene una la misión de cristalizar como espacio de equilibrio y contrapeso. El desarrollo de la neuroética permitirá la enunciación y delimitación de las garantías que actuarán como diques éticos acotando los progresos neurotecnológicos y neurobiológicos. La neurociencia está llamada a enriquecer las estructuras jurídicas formales y, recíprocamente, el neuroderecho integrará los límites y contenidos de los derechos fundamentales en juego con los avances neurocientíficos. Es la actualización del debate dialéctico atemporal entre bioética y bioderecho.

\section{BIBLIOGRAFÍA}

CAPÓ, M. (coord.). «Neuroética. Derecho y Neurociencia», Ludus Vitalis, vol. XIV n. ${ }^{\circ}$ 25, 2006, Laboratorio de Sistemática Humana, Universidad de las Islas Baleares, Palma de Mallorca.

CARRARA, A. «Neuroderecho, entendiendo la mente de los criminales», 2010.

CONILL, J. Ética Hermenéutica, Ed. Tecnos, Madrid, 2006.

CORTINA, A. Neuroética y Neuropolítica: Sugerencias para la Educación Moral, Madrid, Ed. Tecnos, 2011.

- «Neuroética: ¿las bases cerebrales de una ética universal con relevancia política?», ISEGORÍA. Revista de Filosofía Moral y Política n. ${ }^{\circ} 42,2010$.

DEMETRIO CRESPO, E. «Libertad de Voluntad, Investigación sobre el Cerebro y Responsabilidad Penal: aproximación a los fundamentos del moderno debate entre Neurociencias y Derecho Penal», InDret, n. ${ }^{\circ} 2 / 2011$.

- «Neurociencias y Derecho Penal: Nuevas perspectivas en el ámbito de la culpabilidad y tratamiento jurídico-penal de la peligrosidad», 2013.

FARAH, M. «Emerging Ethical Issues in Neuroscience», Nature Neuroscience, n. ${ }^{\circ}$ 5, 2002.

- «Neuroethics: the practical and the philosophical», Trends in Cognitive Science, n. . 9, 2005.

- «Social, Legal and Ethical Implications of Cognitive Neuroscience: "Neuroethics» for Short», Journal of Cognitive Neuroscience, n. ${ }^{\circ} 19,2007$. 
FEIJOO SÁNCHEZ, B. «Derecho Penal y Neurociencias. ¿Una relación tormentosa?» InDret n. ${ }^{\circ}$ 2/11.

GAZZANIGA, M. S. El cerebro ético, Ed. Paidós, Barcelona, 2006.

GREELY, H. T. «Neuroscience and Criminal Justice: Not Responsibility but Treatment», Kansas Law Review n. ${ }^{\circ}$ 56, 2008.

GREENE, J. D. (coord.). «From Neural «Is» To Moral «Ought». What Are The Moral Implications of Neuroscientific Psychology?», GLANNON, W., (Ed.) Defining Right and Wrong in Brain Science, Nature Reviews Neuroscience, Vol. 4, 2003, New York, Ed. DANA Press.

ILLES, J; y BIRD, S. J. «A Modern Context for Ethics in Neuroscience», Trends in Neuroscience n. 29.

KNUTSON, B. y COOPER, J. C. «Functional Magnetic Resonance Imaging of Reward Prediction", Current Opinion in Neurology (Manuscrip N. ${ }^{\circ}$ 18422), 2000.

LEVY, N. Neuroethics. Challenges for the 21st Century, Cambridge University Press, New York (2007).

LOFTUS, E. «Our Changeable memories: Legal and Practical Implications» Nature Reviews / Neuroscience, Vol. 4 (marzo), 2003.

MARCUS, S. J. Neuroethics. Mapping the Field, The Dana Press, New York 2002.

MORA, F. «El Cerebro humano: desafíos para el siglo XXI», Eidon n. ${ }^{\circ} 33,2010$.

MORENO, J. D. «Neuroethics: An Agenda for Neuroscience and Society», Nature Reviews in Neuroscience n. ${ }^{\circ}$ 4, 2003.

- Mind Wars. Brain Research and National Defense, The Dana Press, New York, 2006.

MORSE, S. J. «New Neuroscience, Old Problems: Legal Implications of the Brain Science», Cerebrum n. ${ }^{\circ}$ 6, 2004.

NARVÁEZ MORA, M. "Notas sobre neurociencia, filosofía y derecho», en Legal Theory and Philosophy, Working Papers Series n. ${ }^{\circ} 10$, Universitat de Girona.

PÉREZ MANZANO, M. «Fundamento y Fines del Derecho Penal. Una revisión a la luz de las aportaciones de la Neurociencia», InDret n. ${ }^{\circ} 2 / 11$. 
RODRÍGUEZ, P. «La neurociencia como defensa penal» en la sección Ciencia y Futuro de la edición del diario $A B C$ de 12 de marzo de 2007.

ROOSKIES, A.L. "Neuroethics for the new millenium», Neuron, n. ${ }^{\circ} 35,2002$.

ROSE, S. The Future of the Brain: The Promise and Perils of Tomorrow's Neuroscience, Oxford University Press, Oxford, 2005.

SÁNCHEZ-MIGALLÓN, S. «La ambigüedad de la neuroética», Murcia, 2011 [conferencia pronunciada el 21 de enero de 2011, publicado por la Fundación Bioética en la dirección http://www.bioeticas.org/?dst=neuroetica].

SÁNCHEZ-MIGALLÓN GRANADOS, S. y GIMÉNEZ AMAYA, J. M. «Neuroética» en Fernández Labastida, F. y Mercado, J. A. (editores) Philosophica: Enciclopedia filosófica on line: SÁNCHEZ-MIGALLÓN GRANADOS, S. y GIMÉNEZ AMAYA, J. M. «Neuroética» en FERNÁNDEZ LABASTIDA, F. y MERCADO, J. A. (editores). Philosophica: Enciclopedia filosófica on line: http://www.philosophica. info/archivo/20092008/voces/neuroetica/Neuroetica.html.

- De la Neurociencia a la Neuroética. Narrativa científica y reflexión filosófica, EUNSA, Pamplona, 2010.

SLACHEVSKY, A. "La neuroética: ¿Un neologismo infundado o una nueva disciplina?» Revista Chilena de Neuro-Psiquiatría, v. 45, 1, 2007, págs. 12-15. 
